\title{
Noncausality and Asset Pricing
}

\author{
Matthijs Lof \\ University of Helsinki and HECER
}

Discussion Paper No. 323

April 2011

ISSN 1795-0562

HECER - Helsinki Center of Economic Research, P.O. Box 17 (Arkadiankatu 7), FI-00014 University of Helsinki, FINLAND, Tel +358-9-191-28780, Fax +358-9-191-28781, E-mail info-hecer@helsinki.fi, Internet www.hecer.fi 


\title{
Noncausality and Asset Pricing*
}

\begin{abstract}
Misspecification of agents' information sets or expectation formation mechanisms may lead to noncausal autoregressive representations of asset prices. Annual US stock prices are found to be noncausal, implying that agents' expectations are not revealed to an outside observer such as an econometrician observing only realized market data. A simulation study shows that noncausal processes can be generated by asset-pricing models featuring heterogeneous expectations.
\end{abstract}

JEL Classification: C58, D84, G12, G17

Keywords: noncausal autoregressions, stock prices, heterogeneous expectations.

Matthijs Lof

Department of Political and Economic Studies

University of Helsinki

P.O. Box 17 (Arkadiankatu 7)

FI-00014 University of Helsinki

FINLAND

e-mail: matthijs.lof@helsinki.fi

* I thank Katja Ahoniemi, Markku Lanne, Henri Nyberg and Pentti Saikkonen for helpful comments. The OP-Pohjola Group Research Foundation is gratefully acknowledged for financial support. 


\section{Introduction}

Recent research (e.g. Lanne and Saikkonen, 2011a,b) finds that many financial and economic variables are noncausal, in the sense that current observations seem to depend on both past and future realizations, rather than only on past realizations. This paper discusses noncausality of asset prices and dividends. Recent literature dealing with noncausality focuses mainly on econometric issues, such as instrument selection in GMM estimation (Lanne and Saikkonen, 2011a) and forecasting (Lanne et al., 2010, 2011). In this paper the focus is not on empirical implications but rather on the economic interpretation of noncausality. I show by simulation that noncausality can be generated by excluding relevant information from the econometric model. Asset prices are shown to be noncausal when the econometric model is based on observed market data, but fails to include the correct expectation formation mechanism.

A noncausal autoregressive (AR) process differs from a conventional causal AR process in the dependence on both future and past errors, implying that future errors are predictable given the realized observations of the variable in question. An early discussion of noncausal autoregressions is provided by Breidt et al. (1991). Recently, Lanne and Saikkonen (2011b) introduced a useful reparametrization of the noncausal AR process that allows for explicit dependence on both leads and lags of the variable in question. A stationary noncausal $\operatorname{AR}(r, s)$ process $y_{t}$, depending on $r$ lags and $s$ leads, is defined by:

$$
\phi(L) \varphi\left(L^{-1}\right) y_{t}=\varepsilon_{t}
$$

with $\phi(L)=1-\phi_{1} L-\ldots \phi_{r} L^{r}, \varphi\left(L^{-1}\right)=1-\varphi_{1} L^{-1}-\ldots \varphi_{r} L^{-s}, \varepsilon_{t} \sim$ i.i.d. $\left(0, \sigma^{2}\right)$ and $L$ is a standard lag operator $\left(L^{k} y_{t}=y_{t-k}\right)$. Both polynomials have their roots outside the unit circle. If $\varphi_{j} \neq 0$, for some $j \in\{1, . ., s\}$, (1) is a noncausal process, which may be referred to as purely noncausal if $\phi_{1}=\ldots=\phi_{p}=0$. When $y_{t}$ is a vector, (1) defines a noncausal vector autoregressive process $\operatorname{VAR}(r, s)$ (Lanne and Saikkonen, 2009).

A defining property of a noncausal AR process is its moving average (MA) representation. It is 
well known that any stationary causal $\mathrm{AR}(r, 0)$ process has a backward-looking MA representation:

$$
y_{t}=\phi(L)^{-1} \varepsilon_{t}=\sum_{j=0}^{\infty} \psi_{j} \varepsilon_{t-j}
$$

The MA representation of a noncausal $\operatorname{AR}(r, s)$ process is, on the other hand, both backward- and forward-looking:

$$
y_{t}=\varphi\left(L^{-1}\right)^{-1} \phi(L)^{-1} \varepsilon_{t}=\sum_{j=-\infty}^{\infty} \psi_{j} \varepsilon_{t-j}
$$

while a purely noncausal $\operatorname{AR}(0, s)$ process even has a purely forward-looking MA representation:

$$
y_{t}=\varphi\left(L^{-1}\right)^{-1} \varepsilon_{t}=\sum_{j=0}^{\infty} \psi_{j} \varepsilon_{t+j}
$$

A noncausal process can not be inverted into a backward-looking MA representation, meaning that the errors are nonfundamental. Nonfundamentalness arises when the agents in the economy base their expectations on a larger information set than the information set available to an econometrician, in which case the residuals from the estimated autoregression are not an interpretable function of the true shocks to the agents' information (Hansen and Sargent, 1991; Alessi et al., 2011). In this situation, a noncausal autoregression may fit the data better, because it takes the omitted information into account, by allowing for predictable errors, even without explicit specification of the correct information set (Lanne and Saikkonen, 2011b).

The agents' information set is a flexible concept. The most obvious example of an econometrician having a smaller information set than the agents in the economy is the omission of one or more relevant decision variables from the estimated model. In this paper, I argue that another example of such a situation occurs when the econometrician and the agents observe the same variables, but the econometrician misunderstands the complexity of the expectation formation mechanism, by estimating a linear model while the true mechanism is nonlinear. In section 3, I show that noncausality is often observed when a linear univariate autoregressive model is estimated for a variable that was actually generated by a multivariate or nonlinear process. 
In section 4, the existence of heterogeneous beliefs is shown to be a possible source of noncausality of asset prices. In this case, different agents form different expectations about the future, making it difficult for an econometrician to observe or infer these expectations. This is an important missing piece of information, since on financial markets these expectations ultimately drive asset prices.

To motivate the search for sources of noncausality in asset pricing, the next section presents empirical evidence that historical US stock prices are indeed noncausal.

\section{Empirical results}

To determine whether a causal or noncausal autoregression fits a certain variable $y_{t}$ better, I will follow the model selection procedure proposed by Lanne and Saikkonen (2011b). First, a causal autoregression $\operatorname{AR}(p)$ is estimated by least squares to find the optimal number of lags $p$ such that the model seems adequate in describing the autocorrelation. In this paper the number of lags is selected by the Bayesian Information Criterion (BIC). Next, model (1) is estimated by maximum likelihood for all possible combinations of $r$ and $s$ for which $r+s=p$. After estimating all possible $\operatorname{AR}(r, s)$ models, the specification yielding the largest value of the likelihood function is chosen as the adequate autoregression. If for this model $s>0$, the variable $y_{t}$ is referred to as noncausal. Since causal and noncausal autoregressive processes are indistinguishable when the error terms are Gaussian, another distribution needs to be assumed (Breidt et al., 1991). With macro-economic and financial time series this does not need to be a problem, since Gaussianity if often rejected for these time series due to fat tails. Therefore, t-distributed errors are assumed. Details on maximum likelihood estimation of the noncausal autoregressive model with t-distributed errors are provided by Lanne and Saikkonen (2009, 2011b) for both univariate and multivariate processes.

This model selection procedure is applied to univariate and bivariate time series related to asset pricing, using long-term data on the US stock market provided by Shiller (2005). This dataset includes annual observations from 1871 to 2010 on the value of the S\&P500 index $\left(P_{t}\right)$ and the 
average dividends $\left(D_{t}\right)$ paid to investors holding shares in this index. Noncausality is checked for the log-difference of prices $\left(\triangle p_{t}=\log \left(P_{t}\right)-\log \left(P_{t-1}\right)\right)$ and dividends $\left(\triangle d_{t}=\log \left(D_{t}\right)-\log \left(D_{t-1}\right)\right)$, as well as for the bivariate processes $\left(\triangle p_{t}, \triangle d_{t}\right)^{\prime}$ and $\left(\delta_{t}, \triangle d_{t}\right)^{\prime}$, with $\delta_{t}=\log \left(P_{t} / D_{t}\right)$ is the log pricedividend (PD) ratio. Table 1 depicts the log-likelihood values for all estimated $\operatorname{AR}(r, s)$ models. Log-differenced dividends are found to be causal, but log-differenced prices and both VARs are best described by noncausal processes.

\section{TABLE 1}

\begin{tabular}{|c|c|c|c|c|c|c|c|c|}
\hline & \multicolumn{2}{|c|}{$\Delta p_{t}$} & \multicolumn{2}{|c|}{$\triangle d_{t}$} & \multicolumn{2}{|c|}{$\left(\delta_{t}, \triangle d_{t}\right)^{\prime}$} & \multicolumn{2}{|c|}{$\left(\triangle p_{t}, \triangle d_{t}\right)^{\prime}$} \\
\hline & $(r, s)$ & $\mathscr{L}$ & $(r, s)$ & $\mathscr{L}$ & $(r, s)$ & $\mathscr{L}$ & $(r, s)$ & $\mathscr{L}$ \\
\hline & $(1,0)$ & 41.8 & $(1,0)$ & 123.3 & $(2,0)$ & -240 & $(1,0)$ & -360 \\
\hline \multirow{2}{*}{\multicolumn{2}{|c|}{$(0,1)$}} & 42.8 & $(0,1)$ & 119.9 & $(1,1)$ & -228 & $(0,1)$ & -350 \\
\hline & & & & & $(0,2)$ & -229 & & \\
\hline JB & \multicolumn{2}{|c|}{0.01} & \multicolumn{2}{|c|}{0.00} & \multicolumn{2}{|c|}{0.00} & \multicolumn{2}{|c|}{0.00} \\
\hline LB & \multicolumn{2}{|c|}{0.08} & \multicolumn{2}{|c|}{0.20} & 0.19 & 0.22 & 0.13 & 0.27 \\
\hline MLL & \multicolumn{2}{|c|}{0.36} & \multicolumn{2}{|c|}{0.12} & 0.11 & 0.06 & 0.08 & 0.06 \\
\hline
\end{tabular}

Notes: Log-likelihood values for all possible $\operatorname{AR}(r, s)$ specifications such that $p=r+s$. The specification that maximizes the log-likelihood for each variable is depicted in bold. The lag length $p$ is selected by the BIC, based on a causal Gaussian AR, after which Gaussianity of the residuals is tested with a Jarque-Bera test. JB refers to the $\mathrm{p}$-value of this test. LB and MLL refer to the p-values of the Ljung-Box and McLeod-Li tests ( 5 lags), applied to the residuals of the optimal (non)causal t-distributed AR.

Table 1 further shows some diagnostic test results. After selecting the number of lags $p$ based on a Gaussian causal AR, Gaussianity of the residuals is tested. Gaussianity is rejected by a Jarque-Bera test for all ARs, justifying estimation by non-Gaussian maximum likelihood. The residuals of the autoregression selected as adequate are furthermore subjected to tests for autocorrelation (Ljung-Box) and conditional heteroscedasticity (McLeod-Li). There is no evidence for remaining autocorrelation or heteroscedasticity at the $5 \%$ level. In general, the selected noncausal autoregressions seem to describe these time series well.

The VAR including PD ratios and dividends $\left(\delta_{t}, \triangle d_{t}\right)^{\prime}$ was proposed by Campbell and Shiller (1988) to model agents' expectations of PD ratios and dividends under constant discount rates. The 
result that $\left(\delta_{t}, \triangle d_{t}\right)^{\prime}$ is noncausal is consistent with findings by Lanne and Saikkonen (2009), who show that the VAR proposed by Campbell and Shiller (1987) to model the expected term spread of interest rates is also noncausal. Noncausality of $\left(\delta_{t}, \triangle d_{t}\right)^{\prime}$ implies that agents do not base their expectations only on lags of the PD ratio and the dividend growth rate. The same argument applies to the second VAR in Table 1, including the growth rates of prices and dividends $\left(\triangle p_{t}, \triangle d_{t}\right)^{\prime}$. Taking expectations conditional on all information dated $t-1$ and earlier shows that these expectations can not be expressed as a function of observable data alone:

$$
\begin{aligned}
& E_{t-1}\left[\begin{array}{c}
\delta_{t} \\
\triangle d_{t}
\end{array}\right]=\Phi_{1}\left[\begin{array}{c}
\delta_{t-1} \\
\Delta d_{t-1}
\end{array}\right]+\Pi_{1} E_{t-1}\left[\begin{array}{c}
\delta_{t+1} \\
\Delta d_{t+1}
\end{array}\right]+E_{t-1}\left[\begin{array}{c}
\varepsilon_{1, t} \\
\varepsilon_{2, t}
\end{array}\right] \\
& E_{t-1}\left[\begin{array}{c}
\triangle p_{t} \\
\triangle d_{t}
\end{array}\right]=\Pi_{1} E_{t-1}\left[\begin{array}{c}
\triangle p_{t+1} \\
\triangle d_{t+1}
\end{array}\right]+E_{t-1}\left[\begin{array}{c}
\varepsilon_{1, t} \\
\varepsilon_{2, t}
\end{array}\right] .
\end{aligned}
$$

An economic interpretation of noncausality is therefore that agents' expectations are not revealed when only realized prices and dividends are observed. Future realizations or a wider information set are required to infer the true expectations. This observed dependence on leading observations may be caused by misspecification of the agents' information set. This issue is further discussed in the remainder of this paper.

\section{Misspecified autoregressions}

I illustrate that misspecification of the econometric model can cause noncausality by simulating two simple AR processes. In the first example the variable of interest is generated by a multivariate process, but estimated as a univariate process. In the second example the data generating process is nonlinear, while a linear model is estimated.

First, the omitted-variable problem is considered. The data are generated by a first order causal 
bivariate process:

$$
\left[\begin{array}{l}
x_{t} \\
y_{t}
\end{array}\right]=\left[\begin{array}{ll}
a & b \\
0 & c
\end{array}\right]\left[\begin{array}{c}
x_{t-1} \\
y_{t-1}
\end{array}\right]+\left[\begin{array}{c}
\varepsilon_{x, t} \\
\varepsilon_{y, t}
\end{array}\right] \varepsilon_{x, t}, \varepsilon_{y, t} \sim t_{3}(0,1) .
$$

The errors $\varepsilon_{x, t}$ and $\varepsilon_{y, t}$ are independent and t-distributed with three degrees of freedom, zero mean and variance one. I calibrate $a=c=0.8$ and generate 200 observations of $x_{t}$ and $y_{t}$ for different values of $b$.After this simulation, $y_{t}$ is dropped from the information set and $x_{t}$ is estimated as a univariate AR process to check noncausality by the model selection procedure discussed in the previous section. This simulation is repeated 5000 times. Table 2 shows how often the model selection procedure selects causal and noncausal representations for different values of $b .{ }^{1}$ When $b=0$, the causal autoregression is the correct specification and is selected in $98 \%$ of the simulations. However, when $b \neq 0, x_{t}$ is driven by two shocks $\varepsilon_{x, t}$ and $\varepsilon_{y, t}$, while only one shock can be identified by estimating an autoregression. Due to this nonfundamentalness, a noncausal autoregression is selected as the adequate specification more often, up to $40 \%$ of the simulations for $b=0.8$. Interestingly, when $b$ becomes larger in absolute value, $\varepsilon_{y, t}$ becomes the dominant shock and the causal AR is again selected more often. In the case that $b=10$, the contribution of $\varepsilon_{x, t}$ to the dynamics of $x_{t}$, relative to the contribution of $\varepsilon_{y, t}$, is so small that the true process can be well approximated by a causal AR process with only one shock.

\section{TABLE 2}

\begin{tabular}{lccccccccc}
\hline \hline$b$ & -10 & -0.5 & 0 & 0.2 & 0.5 & 0.8 & 1 & 2 & 10 \\
\hline Causal & $93 \%$ & $68 \%$ & $98 \%$ & $94 \%$ & $69 \%$ & $60 \%$ & $66 \%$ & $87 \%$ & $93 \%$ \\
Noncausal & $7 \%$ & $32 \%$ & $2 \%$ & $6 \%$ & $31 \%$ & $40 \%$ & $34 \%$ & $13 \%$ & $7 \%$
\end{tabular}

Notes: Percentage of causal and noncausal outcomes of the AR for $x_{t}$ after 5000 simulations of model (2), with $a=c=0.8$ and different values of $b$. The sample size in each simulation is 200 observations.

\footnotetext{
${ }^{1}$ The simulations are also carried out for different values of $a$ and $c$ between -1 and 1 and for different sample sizes (500 and 1000). As long as $a$ and $c$ are not too close to zero, (i.e. the simulated data are not white noise), the results are similar to those in Table 2 and are therefore not explicitly reported.
} 
Next, a univariate nonlinear Logistic Smooth Transition Autoregressive (LSTAR) process is generated:

$$
\begin{gathered}
y_{t}=\alpha_{1} y_{t-1}\left(1-G\left(s_{t-1}\right)\right)+\alpha_{2} y_{t-1} G\left(s_{t-1}\right)+\varepsilon_{t} \\
G\left(s_{t-1}\right)=\left(1+\exp \left[-\gamma s_{t-1}\right]\right)^{-1} \\
\varepsilon_{t} \sim t_{3}(0,1) .
\end{gathered}
$$

This process is a weighted average of two causal AR(1) regimes. Since the weights are time-varying, the process is nonlinear. However when $\gamma=0$, the transition function $G\left(s_{t-1}\right)=1 / 2$ in all periods, so the process is linear. On the other hand, when $\gamma=\infty, G\left(s_{t-1}\right)$ is either zero or one, meaning the process reduces to a Threshold Autoregressive (TAR) process. In short, the process becomes more nonlinear when $\gamma$ increases. I choose the transition variable $s_{t-1}=\Delta y_{t-1}$ and the calibration $\alpha_{1}=0.8$ and $\alpha_{2}=-0.2$, so that each regime is stationary and differs considerably from the other regime. A sample of 200 observations is simulated for different values of $\gamma: 0,0.2,0.5,1,2$ and $10.000(\approx \infty)$, after which a linear AR model is fitted to the data to check for noncausality. Table 3 displays the results of 5000 repetitions. In the linear case $(\gamma=0)$, a noncausal specification is selected in $4 \%$ of the simulations. However, the number of noncausal representations selected steadily increases with $\gamma$, up to $66 \%$ of the simulations for the TAR model. These results show that not only after omitted variables, but also after misspecification of the functional form, a noncausal process often approximates the true process better than a causal process, even if these processes depend by no means on the future.

\section{TABLE 3}

\begin{tabular}{lcccccc}
\hline \hline$\gamma$ & 0 & 0.2 & 0.5 & 1 & 2 & $\infty$ \\
\hline Causal & $95 \%$ & $92 \%$ & $82 \%$ & $68 \%$ & $53 \%$ & $34 \%$ \\
Noncausal & $4 \%$ & $8 \%$ & $18 \%$ & $33 \%$ & $47 \%$ & $66 \%$
\end{tabular}

Notes: Percentage of causal and noncausal outcomes of the AR for $y_{t}$ after 5000 simulations of model (3), with $s_{t-1}=\triangle y_{t-1}, \alpha_{1}=0.8, \alpha_{2}=-0.2$. The sample size in each simulation is 200 observations. 


\section{Heterogeneous expectations}

Returning to asset pricing, the results of the previous section suggest that the observed noncausality in Table 1 could be the result of the misspecification of agents' expectation formation mechanisms: Agents participating in the stock market base their expectations on more information than past prices and dividends alone and/or their expectations are a nonlinear function of the available information.

The existence of heterogeneous beliefs is a natural candidate for such a situation. Kasa et al. (2010) derive conditions under which informational heterogeneity (agents receiving different signals about future dividends) imposes agents to forecast the forecasts of other agents, as in Townsend (1983), which leads to a nonrevealing equilibrium. Kasa et al. (2010) explicitly show how the process of prices and dividends is under these conditions not invertible into a backward-looking moving average process (i.e. is nonfundamental) and argue that an econometrician who does not observe these different signals will misinterpret the residuals from a VAR as shocks to the agents' information.

To check what type of investor behavior generates noncausality, I simulate asset prices under different expectation regimes. I consider a representative-agent model and two models featuring boundedly rational agents with heterogeneous beliefs. After each simulation, I act as an econometrician who does not understand the structure of the underlying model and estimate both causal and noncausal VARs for prices and dividends, to find out which VAR fits the data best. The starting point for this simulation exercise are the dividends, which are assumed to be exogenous, not depending on asset prices. To be precise, dividends are generated by a causal AR(1) process:

$$
d_{t}=\alpha_{1}+\alpha_{2} d_{t-1}+\varepsilon_{t}
$$

with $\varepsilon_{t} \sim t_{3}\left(0, \sigma_{\varepsilon}^{2}\right)$. The fundamental value $p_{t}^{*}$ of the asset equals the sum of all expected future dividends, discounted at a constant discount factor $r$ : 


$$
\begin{aligned}
p_{t}^{*} & =\sum_{i=0}^{\infty} \frac{E_{t-1}\left[d_{t+i}\right]}{(1+r)^{i}} \\
E_{t-1}\left[d_{t+i}\right] & =\alpha_{1}+\alpha_{2} E_{t-1}\left[d_{t+i-1}\right] .
\end{aligned}
$$

In a world where all agents have rational and homogeneous beliefs about the future (i.e. a rational representative-agent model) the asset price should reflect the expected fundamental value of the asset:

$$
p_{t}=p_{t}^{*}+\eta_{t} \quad \eta_{t} \sim t_{3}\left(0, \sigma_{\eta}^{2}\right)
$$

The i.i.d. error term $\eta_{t}$ is added so that $p_{t}$ is not an exact linear function of $d_{t-1}$, which would make the parameters in a VAR including prices and dividends not identifiable. The error term can however be justified as noise due to frictions, the existence of noise traders or minor uncertainties about fundamentals.

A more general model relaxes the assumptions of homogeneity and rationality and allows for heterogeneous beliefs. I follow the asset-pricing model proposed by Brock and Hommes (1998), featuring many types of boundedly rational agents who form different beliefs about the future. With $H$ different types of agents, asset prices are determined by the following equation:

$$
p_{t}=\sum_{h=1}^{H} \frac{n_{h, t} E_{h, t-1}\left[p_{t+1}+d_{t+1}\right]}{1+r}+\eta_{t}
$$

where $E_{h, t}(\cdot)$ represents the expectation formation mechanism of agent type $h$ and $n_{h, t}$ is the fraction of the population behaving according to type $h$ at time $t$. In the special case that $H=1$ and $E_{1, t}(\cdot)$ denotes rational expectations $E_{t}(\cdot),(7)$ reduces to (6). To introduce heterogeneous beliefs it is useful to formulate (7) in deviation from the fundamental value:

$$
x_{t}=\sum_{h=1}^{H} \frac{n_{h, t} f_{h, t}}{1+r}+\eta_{t}
$$

with $x_{t}=p_{t}-p_{t}^{*}$ is the realized difference from the fundamental value and $f_{h, t}=E_{h, t-1}\left[p_{t+1}\right]-$ $E_{t-1}\left[p_{t+1}^{*}\right]$. Following Brock and Hommes (1998), agents hold identical beliefs about the funda- 
mental value, but disagree on the dynamics of the deviation from the fundamental value. In particular, each type applies linear prediction rules based on lagged prices to form their expectations:

$$
f_{h, t}=g_{h} x_{t-1}+b_{h}
$$

The fraction of each type, $n_{h, t}$, varies over time according to evolutionary dynamics. The type of agent that realizes a high profit from trading in the previous period will become more influential in the next period:

$$
n_{h, t}=\frac{\exp \left(\beta U_{h, t-1}\right)}{\sum_{i=1}^{H} \exp \left(\beta U_{i, t-1}\right)},
$$

where $U_{h, t}=\left(x_{t}-(1+r) x_{t-1}\right)\left(f_{h, t-1}-(1+r) x_{t-2}\right)-c_{h}$ denote the realized profits for each type, such that the fractions of all types add up to one. A full derivation of these equations is provided in Brock and Hommes (1998). These evolutionary dynamics are comparable to the 'forecasting the forecasts of others' property considered by Townsend (1983) and Kasa et al. (2010): Agents do not commit only to their own beliefs, but take into consideration the expectations of other agents, knowing that the expectations of others have a direct effect on asset prices. The parameter $\beta$ defines the willingness or capability of agents to switch to another strategy. I now consider an example with two different agent types: The first type is the fundamentalist, who believes deviations from the fundamental value should disappear:

$$
f_{F, t}=0 \text {. }
$$

The other type is the chartist or trend-follower, who believes deviations from the fundamental value in the previous period will persist:

$$
f_{C, t}=g_{C} x_{t-1}
$$

The parameter $g_{C}$ defines the difference between the behavior of the agents. When $g_{C}=0$, both types are identical. When $0<g_{C}<1+r$, both types agree that deviations from the fundamental 
value should disappear over time, but they disagree about the pace of this correction. In Brock and Hommes (1998) and in this paper $g_{C} \geq 1+r$, meaning the chartists believe that the asset price will diverge from the fundamental value. Fundamentalists will therefore buy stocks when the price is under its fundamental valuation and sell when it is above. Chartists act the other way around which may create both positive and negative stock price bubbles even in the absence of random shocks (Brock and Hommes, 1998). Chartists are commonly thought of as technical traders, although Parke and Waters (2007) argue that similar behavior could be observed when agents experiment with different information sets to form expectations. The representative-agent benchmark (6) is nested in the model with fundamentalists and chartists (7-12): The models can be made identical either by setting $g_{C}=0$, or $n_{F, t}=1 \forall t$.

A final example includes two other types: Optimists and pessimists (or bulls and bears). The optimist type forms expectations with a positive bias, while the pessimist type forms expectations with a negative bias:

$$
\begin{aligned}
f_{O, t} & =b \\
f_{P, t} & =-b,
\end{aligned}
$$

with $b \geq 0$. This model is identical to the representative-agent benchmark (6) if $b=0$. Optimists believe the asset is undervalued while pessimists believe the asset is overvalued. This disagreement could be the result of heterogeneous information on the fundamentals: The optimists (pessimist) receives positive (negative) signals about future fundamentals, although also other factors such as different levels of risk-aversion could cause the different beliefs.

I simulate dividends (4) and asset prices according to the representative-agent model (6), the fundamentalist-chartist model (7-12) and the optimist-pessimist model (7-10,13). Plots of 200 simulated observations of the asset prices under each regime are given in Figure 1, together with the calibration of the parameters. The calibration of the profit functions and switching probabilities (10) as well as the behavioral parameters of the chartists and fundamentalists is identical to the calibration by Brock and Hommes (1998). The bias in the optimistic and pessimistic beliefs is calibrated at 
$2.5 \%$ of the average fundamental value. Figure 1 shows that under the representative-agent model, the difference between the fundamental values and the realized price is i.i.d. random noise (top panel). With the fundamentalist-chartist model, longer lasting deviations are observed. Thinking of annual data, the middle panel shows several examples of stock price bubbles lasting up to a decade. Finally, the bottom panel of Figure 1 shows the optimist-pessimist model, with continuous cycles of overvaluation followed by undervaluation lasting just a couple of years.

After each simulation, the model selection procedure described in section 2 is applied to determine whether the VAR including (demeaned) prices and dividends $\left(p_{t}, d_{t}\right)^{\prime}$ is causal or noncausal. Since dividends follow a stationary AR process, there is no need to take $(\log )$ differences. This simulation is repeated 5000 times. Table 4 shows how often causal and noncausal specifications are selected for each model..
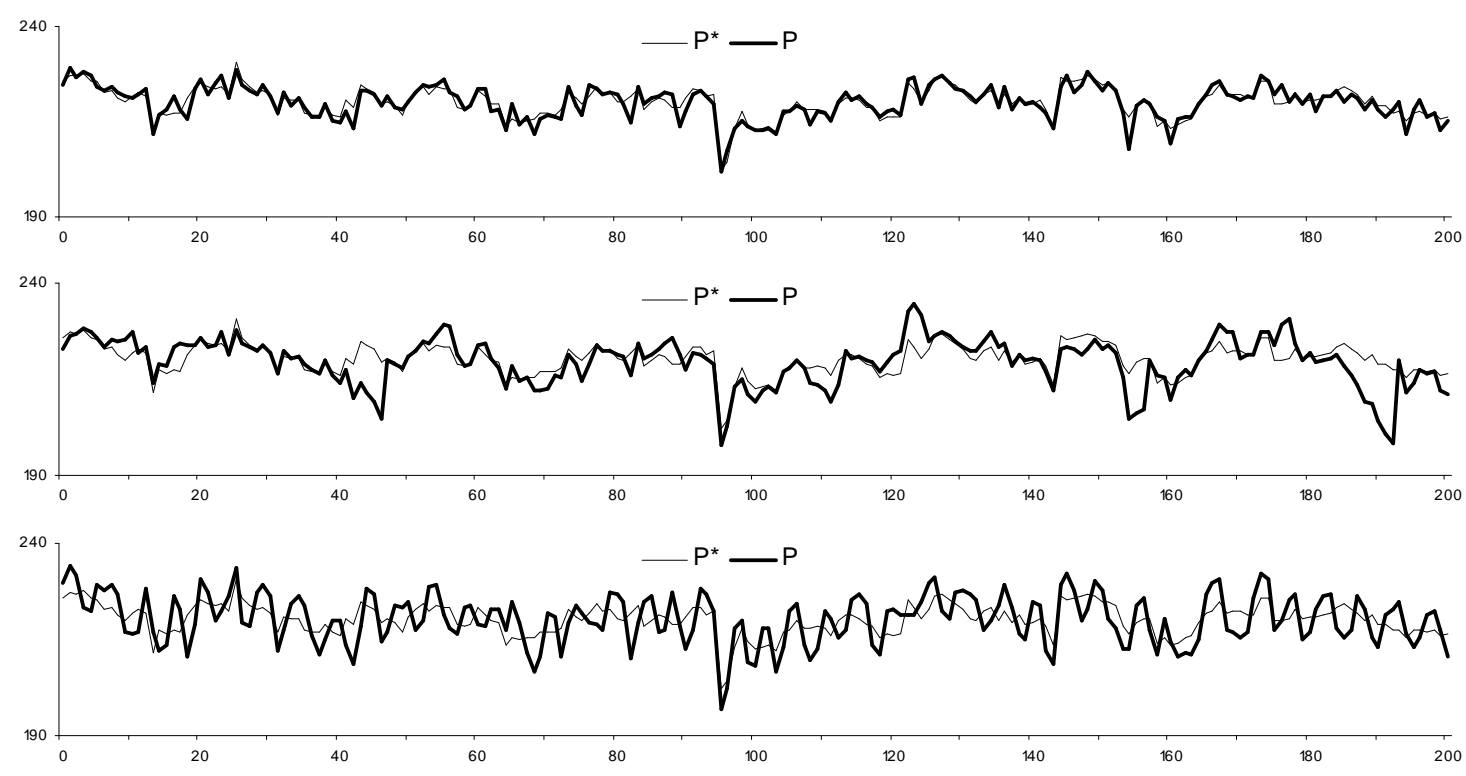

Figure 1: Simulated asset prices. Fundamental values and realized prices generated by: Representative agent (Top panel), Fundamentalists and Chartists (Middle panel) and Optimists and Pessimists (Bottom panel). Calibration: $\alpha_{1}=4, \alpha_{2}=0.8, \sigma_{\varepsilon}^{2}=1, r=0.1, \sigma_{\eta}^{2}=2, \beta=3.6, g^{C}=1.2, c_{F}=1, c_{C}=c_{O}=c_{P}=0$, $b=5.5$ 


\section{TABLE 4}

\begin{tabular}{lccc}
\hline \hline & $\begin{array}{c}\text { Representative } \\
\text { agent }\end{array}$ & $\begin{array}{c}\text { Fundamentalists } \\
\text { and Chartists }\end{array}$ & $\begin{array}{c}\text { Optimists and } \\
\text { Pessimists }\end{array}$ \\
\hline Causal & $98 \%$ & $10 \%$ & $61 \%$ \\
Noncausal & $2 \%$ & $90 \%$ & $40 \%$ \\
\hline
\end{tabular}

Notes: Percentage of causal and noncausal outcomes of the VAR for $\left(p_{t}, d_{t}\right)^{\prime}$ after 5000 simulations with a representative-agent model and with heterogeneous-agents models. The sample size in each simulation is 200 observations.

With a representative agent the VARs of prices and dividends are found to be almost exclusively causal. However, with the optimist-pessimist model noncausality is found for almost $40 \%$ of the simulations and with the fundamentalist-chartist model even in $90 \%$ of the simulations, even though all types of agents considered are fully backward-looking in the sense that they base their decisions only on past prices and dividends. These results confirm that heterogeneous beliefs are a potential source of noncausality. This is consistent with the simulation results in section 3 , since the switching between strategies causes nonlinearity in the prices, even if all beliefs are linear functions of realized data. Moreover, the fractions of each type of agent in the population can also be interpreted as an omitted variable. Parke and Waters (2007) note that asset prices are generated by a process $P_{t}=f\left(\Omega_{t-1}, n_{t}, \varepsilon_{t}\right)$, where $\Omega_{t-1}$ includes all past prices and dividends and $n_{t}$ include the fractions of each type. In this case an econometrician will have access to $\Omega_{t-1}$, but can not observe behavior or expectations. An estimated model will therefore be of the form $P_{t}=\hat{f}\left(\Omega_{t-1}, \hat{\varepsilon}_{t}\right)$, so that $n_{t}$ is an omitted variable.

\section{Conclusions}

This paper presents empirical results confirming that several autoregressions related to asset pricing are noncausal. A simulation study shows that nonlinearity caused by heterogeneous beliefs is a potential source of noncausality. This is an example of the econometrician having a smaller information set available than the actual agents in the economy. When only realized market data are 
observed, an important piece of information about the asset pricing process is omitted, namely the expectations and fractions of each type of agent.

Investor heterogeneity is not the only potential source of noncausality. A representative agent model may also be nonlinear, because of a time-varying (stochastic) discount factor. Moreover, in reality dividends are generated by a more complicated process than an AR(1) process and depend on many other variables. When markets are efficient, agents will rationally use all available information to form expectations about the future, making it nearly impossible for an econometrician to create a dataset that can replicate these expectations. Both these cases could potentially generate noncausal asset prices.

\section{References}

Alessi, L., M. Barigozzi, and M. Capasso: 2011, 'Nonfundamentalness in Structural Econometric Models: A Review'. International Statistical Review (forthcoming).

Breidt, F. J., R. A. Davis, K.-S. Lh, and M. Rosenblatt: 1991, 'Maximum likelihood estimation for noncausal autoregressive processes'. Journal of Multivariate Analysis 36(2), 175-98.

Brock, W. A. and C. H. Hommes: 1998, 'Heterogeneous beliefs and routes to chaos in a simple asset pricing model'. Journal of Economic Dynamics and Control 22(8-9), 1235-1274.

Campbell, J. Y. and R. J. Shiller: 1987, 'Cointegration and Tests of Present Value Models'. Journal of Political Economy 95(5), 1062-88.

Campbell, J. Y. and R. J. Shiller: 1988, 'The Dividend-Price Ratio and Expectations of Future Dividends and Discount Factors'. Review of Financial Studies 1(3), 195-228.

Hansen, L. P. and T. J. Sargent: 1991, 'Two Difficulties in Interpreting Vector Autoregressions'. In: L. P. Hansen and T. J. Sargent (eds.): Rational Expectations Econometrics. Westview Press, Inc., Boulder, CO, pp. 77-119. 
Kasa, K., T. B. Walker, and C. H. Whiteman: 2010, 'Heterogeneous Beliefs and Tests of Present Value Models'. Unpublished manuscript.

Lanne, M., A. Luoma, and J. Luoto: 2011, 'Bayesian Model Selection and Forecasting in Noncausal Autoregressive Models'. Journal of Applied Econometrics (forthcoming).

Lanne, M., J. Luoto, and P. Saikkonen: 2010, 'Optimal Forecasting of Noncausal Autoregressive Time Series'. HECER Discussion Paper 286.

Lanne, M. and P. Saikkonen: 2009, 'Noncausal vector autoregression'. Research Discussion Papers 18/2009, Bank of Finland.

Lanne, M. and P. Saikkonen: 2011a, 'GMM Estimation with Noncausal Instruments'. Oxford Bulletin of Economics and Statistics (forthcoming).

Lanne, M. and P. Saikkonen: 2011b, 'Noncausal Autoregressions for Economic Time Series'. Journal of Time Series Econometrics (forthcoming).

Parke, W. R. and G. A. Waters: 2007, 'An evolutionary game theory explanation of ARCH effects'. Journal of Economic Dynamics and Control 31(7), 2234-2262.

Shiller, R. J.: 2005, Irrational exuberance. Princeton University Press.

Townsend, R. M.: 1983, 'Forecasting the Forecasts of Others'. Journal of Political Economy 91(4), $546-88$. 